\title{
Lower lip pits with sinus tracts in Van der Woude syndrome: a case report and review of the literature
}

\author{
Jong-Ho Kim, Byungkwon Kang, Baek-Kyu Kim \\ Department of Plastic and Reconstructive Surgery, Seoul National University Bundang Hospital, Seoul National University College of \\ Medicine, Seongnam, Korea
}

In Van der Woude syndrome (VWS), a rare congenital disease, lower lip pits (LLPs) can cause an aesthetically significant deformity. Surgical treatment of LLPs is necessary if they cause recurrent inflammation or aesthetic problems. Intraoperatively, surgeons should keep in mind the possibility of deep extension of the sinus tract and the relative deficiency of the midline in VWS, which increases the risk of lip disfigurement. Herein, we emphasize the importance of using a tissue-preserving technique to improve aesthetic results in VWS patients with a sinus tract.

Keywords Lip / Van der Woude syndrome / Anomalies, multiple / Fistula / Case reports

\author{
Correspondence: Baek-Kyu Kim \\ Department of Plastic and \\ Reconstructive Surgery, Seoul \\ National University Bundang Hospital, \\ Seoul National University College of \\ Medicine, 82 Gumi-ro 173beon-gil, \\ Bundang-gu, Seongnam 13620, Korea \\ Tel: +82-31-787-7225 \\ Fax: +82-31-787-4055 \\ E-mail: plasrecon@gmail.com
}

Received: April 23, 2021 - Revised: August 11, 2021 - Accepted: August 30, 2021

pISSN: 2234-6163 • elSSN: 2234-6171 • https://doi.org/10.5999/aps.2021.00850 • Arch Plast Surg 2022;49:55-58

\section{INTRODUCTION}

Van der Woude syndrome (VWS) is a rare disorder that clinically presents as lower lip pits (LLPs) with or without cleft lip or palate. The pattern of inheritance is autosomal dominant, and the condition was first described by Van der Woude [1]. VWS is recognized as the most common type of syndromic cleft lip, representing $2 \%$ of all cleft lip cases with or without cleft palate [2]. The prevalence is reported as $1 / 75,000$ to $1 / 100,000$, and it is caused by mutations in the gene for interferon regulatory factor 6 (IRF6), which is located in chromosome 1q32-q41 [3]. Many studies have focused on the location or characteristics of LLPs $[4,5]$. However, there are few reports regarding the surgical technique or precautions for LLPs with sinus tracts. Thus, the purpose of this report was to present a rare case of LLPs with long sinus tracts in VWS and share proper surgical techniques.

\section{CASE}

A 9-year-old boy presented to our outpatient clinic with a congenital deformity of the lower lip. On physical examination, we could observe bilateral pits in the vermilion of the lower lip (Fig. 1). With gentle compression, a saliva-like mucous secretion was excreted through the orifices of the lower lip, and the patient had a history of recurrent inflammation on his lower lip. Otherwise, the patient had no specific medical history and family history of other craniofacial deformities. Based on these pathognomonic features, VWS was diagnosed. Under general anesthesia, gentian violet dye was injected into the sinus fistula to trace and remove the entire tract. Meticulous dissection was then performed to remove the entire lining of the tract while preserving surrounding tissues, including the mucosa and orbicularis oris muscle. As intraoperative findings, the depth of the pits was 28 $\mathrm{mm}$ on the right side and $13 \mathrm{~mm}$ on the left side (Fig. 2). The wound was closed in a direction parallel to the vermilion border (Fig. 3). On a histopathologic exam of the specimen, the tract 




Fig. 1. Preoperative photograph showing bilateral paramedian pits of the lower lip in a patient with Van der Woude syndrome.
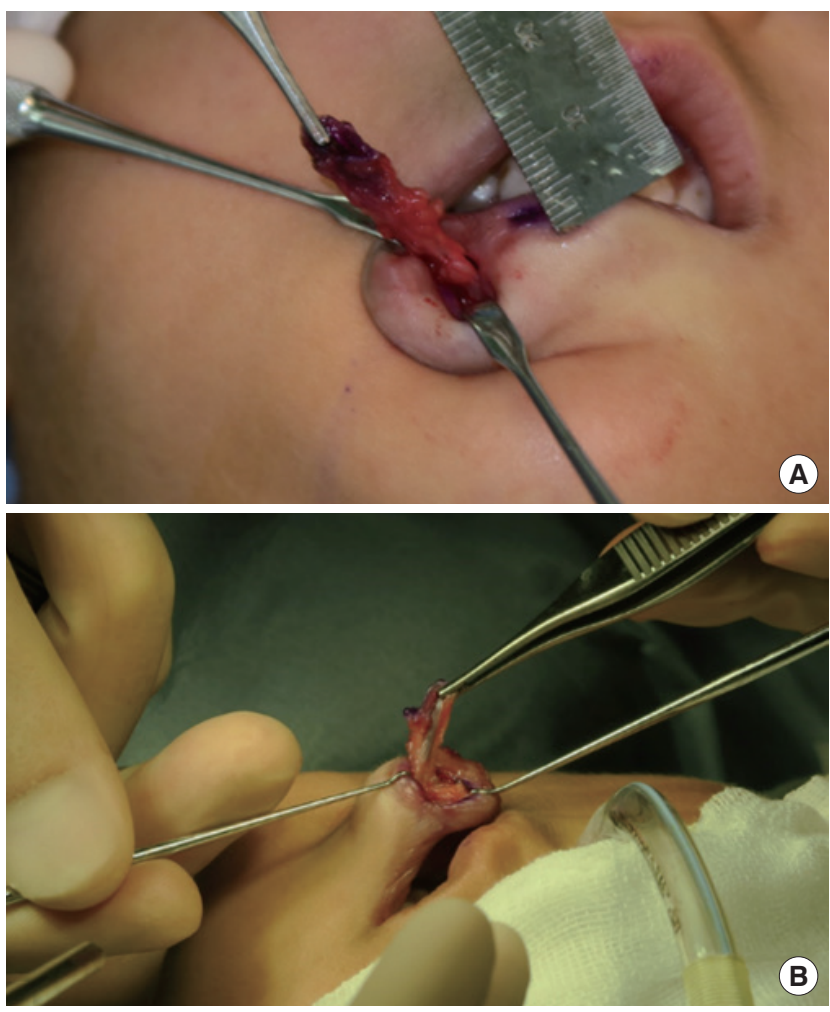

Fig. 2. Intraoperatively, a gentian violet solution was injected to identify the fistulous tracts. (A) On the right side, the length of the tract was $28 \mathrm{~mm}$. (B) On the left side, the length was $13 \mathrm{~mm}$.

lined by squamous epithelium with minor salivary glandular tissue was identified. The patient had no complications, including mucocele formation or recurrence of inflammation, at a 6-month follow-up (Fig. 4).

\section{DISCUSSION}

LLPs occur in more than $90 \%$ of cases of VWS and represent the most important characteristic of this syndrome. If LLPs

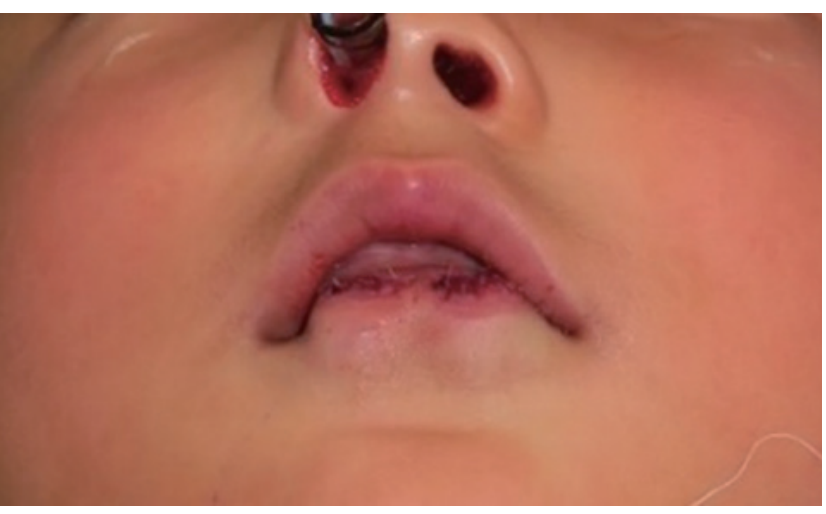

Fig. 3. Postoperative photograph after removal of the sinus tracts.

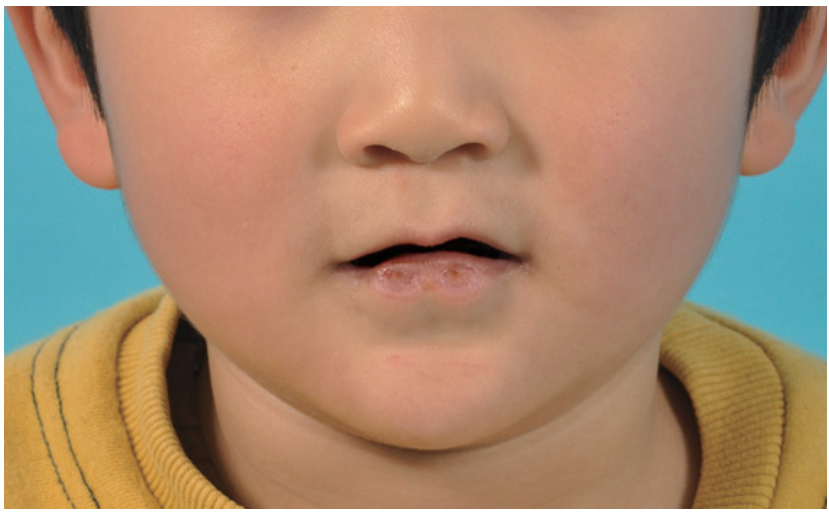

Fig. 4. Six-month postoperative photograph.

cause recurrent inflammation by draining secretions or pose a cosmetic problem, the tract of the pits should be completely resected due to the communication with the underlying salivary glands [6]. Even if LLPs do not result in the above-mentioned problems, chronic secretion of saliva can cause considerable discomfort. The entirety of the tract and its salivary environment must be resected to avoid recurrence and residual mucoceles [7]. Rizos and Spyropoulos [4] reported that a sinus tract could extend up to $2.5 \mathrm{~cm}$ to the sulcus labialis. Peralta-Mamani et al. [8] recently published a systematic review of the treatment of LLP in VWS, and found that the use of a vertical wedge excision and inverted-T lip reduction showed better results than simple excision. Simple excision may result in postoperative complications, such as mucocele and pit recurrence [9]. Other studies have also advocated for the complete excision of LLPs, including the adjacent tissue [10,11]. Mutaf et al. [12] used a split-lip advancement flap to reconstruct the labial tonus and Chen et al. [13] reported better results in their 34 patients with the inverted -T lip reduction technique. We also advocate for using these techniques and support the proposal made in other studies that fusiform excision alone is sufficient for some patients. However, 
in some patients with a long sinus tract, the cosmetic results have not been satisfactory when performing simple or wedge excision. VWS is likely to involve a relative deficiency of the midline, which means that en bloc excision poses the risk of causing a "whistle lip deformity" or another disfigurement in these patients [4]. We emphasize the need for a tissue-preserving technique to improve aesthetic results in VWS patients, especially those who have long sinus tracts. If surgical resection is performed along a longer sinus tract, there could be a higher possibility of tissue insufficiency. It is difficult to effectively preserve mucosal tissue along the sinus tract using the previously introduced methods. In order to obtain better cosmetic results, the remaining tissue should be manipulated after the sinus tracts are excised with tissue preservation. Therefore, mucosal and muscular tissue surrounding the sinus tract should be carefully dissected in order to minimize the loss of tissue while at the same time performing complete surgical removal of the tract. To ensure total resection of the sinus tract, a marker such as methylene blue or gentian violet solution (as used in this case) can be injected into the orifice to mark the sinus tract [14]. In conclusion, although LLPs in VWS give the impression that simple excision would suffice, surgeons should keep in mind the possibility of deep extension of the sinus tract. In these cases, injecting dyes and meticulous dissection to minimize the loss of surrounding tissue can produce improved aesthetic results.

\section{NOTES}

\section{Conflict of interest}

No potential conflict of interest relevant to this article was reported.

\section{Ethical approval}

The study was performed in accordance with the principles of the Declaration of Helsinki. Written informed consent was obtained.

\section{Patient consent}

The patient's guardian provided written informed consent for the publication and the use of his images.

\section{Author contribution}

Conceptualization: JH Kim. Data curation: JH Kim, B Kang. Formal analysis: B Kang. Methodology: JH Kim, BK Kim. Project administration: BK Kim. Visualization: JH Kim, B Kang. Writing-original draft: JH Kim. Writing-review \& editing: JH Kim, B Kang.

\section{ORCID}

Jong-Ho Kim https://orcid.org/0000-0003-0998-6358

Byungkwon Kang https://orcid.org/0000-0002-7704-0786

Baek-Kyu Kim https://orcid.org/0000-0001-9974-5165

\section{REFERENECES}

1. Van der Woude A. Fistula labii inferioris congenita and its association with cleft lip and palate. Am J Hum Genet 1954; 6:244-56.

2. Schutte BC, Basart AM, Watanabe Y, et al. Microdeletions at chromosome bands 1q32-q41 as a cause of Van der Woude syndrome. Am J Med Genet 1999;84:145-50.

3. Shotelersuk V, Srichomthong C, Yoshiura K, et al. A novel mutation, 1234del(C), of the IRF6 in a Thai family with Van der Woude syndrome. Int J Mol Med 2003;11:505-7.

4. Rizos M, Spyropoulos MN. Van der Woude syndrome: a review: cardinal signs, epidemiology, associated features, differential diagnosis, expressivity, genetic counselling and treatment. Eur J Orthod 2004;26:17-24.

5. Kaul B, Mahajan N, Gupta R, et al. The syndrome of pit of the lower lip and its association with cleft palate. Contemp Clin Dent 2014;5:383-5.

6. Kirzioglu Z, Erturk MS. Congenital lower lip pits (Van der Woude syndrome): report of a case.J Contemp Dent Pract 2006; 7:134-40.

7. Brookes JT, Canady JW. Surgical correction of congenital lower lip sinuses in Van der Woude syndrome. Cleft Palate Craniofac J 2007;44:555-7.

8. Peralta-Mamani M, Terrero-Perez A, Dalben G, et al. Treatment of lower lip pits in Van der Woude syndrome: a systematic review. Int J Oral Maxillofac Surg 2018;47:421-7.

9. Bertin H, Diallo-Hornez G, Isidor B, et al. Surgical management of lower lip pits in Van der Woude syndrome. J Stomatol Oral Maxillofac Surg 2018;119:67-70.

10. Richardson S, Khandeparker RV. Management of lip pits in Van der Woude syndrome: a clinical classification with difficulty index. J Oral Maxillofac Surg 2016;74:1849.

11. Ziai MN, Benson AG, Djalilian HR. Congenital lip pits and Van der Woude syndrome. J Craniofac Surg 2005; 16:930-2.

12. Mutaf M, Sensoz O, Ustuner ET. The split-lip advancement technique (SLAT) for the treatment of congenital sinuses of the lower lip. Plast Reconstr Surg 1993;92:615-20.

13. Chen $\mathrm{CH}$, Liao HT, Shyu VB, et al. Inverted-T lip reduction for lower lip repair in Van der Woude syndrome: a review and comparison of aesthetic results. Int J Oral Maxillofac Surg 2013;42:198-203. 
14. Castro CH, De Carvalho MF, Veloso DC, et al. An alternative technique using a gutta percha points and blue methylene to excision of congenital fistula of lower lip in patient with Van der Woude syndrome. Stomatologija 2012;14:60-4. 